\title{
Surgical resection and post-operative radiotherapy in an adult renal neuroblastoma patient with multiple bone and joint metastases: A case report
}

\author{
QIU-LONG LIU ${ }^{1}$, QI-LIAN LIANG ${ }^{1}$, WEN-TING OU ${ }^{1}$, ZHOU-YU LI $^{2}$ and XIANG-NING ZHANG ${ }^{3}$ \\ ${ }^{1}$ Department of Oncology, Affiliated Hospital of Guangdong Medical College, Zhanjiang, Guangdong 524001; \\ ${ }^{2}$ Department of Radiotherapy, Cancer Center of Guangzhou Medical University, Guangzhou, \\ Guangdong 510095; ${ }^{3}$ Department of Pathophysiology, School of Basic Medical Science, \\ Guangdong Medical College, Dongguan, Guangdong 523808, P.R. China
}

Received April 19, 2014; Accepted January 9, 2015

DOI: $10.3892 / 01.2015 .3109$

\begin{abstract}
Renal neuroblastoma is uncommon, particularly in adults, with only a few cases having been reported in studies published in the English language. The incidence is only 0.12 cases $/ 1$ million individuals in those aged $>20$ years. Studies of the pathogenesis, biological characteristics, treatment and prognosis of renal neuroblastoma are limited due to this low incidence. The present study reports the case of a 22-year-old adult female who was diagnosed with a left renal neuroblastoma by computed tomography (CT), bone scan and pathological examination. The patient underwent a left nephroureterectomy, ipsilateral lymph node dissection and post-operative radiotherapy, prior to discharge 60 days after admittance. At the nine-month follow-up examination, the patient showed no evidence of recurrence, progression or metastatic disease on the CT scans of the chest, abdomen and pelvis. Renal neuroblastoma is extremely uncommon in adults. The diagnosis and treatment of renal neuroblastoma is complicated by the overall low incidence, lack of specific treatment guidelines, advanced disease state due to late presentation, and its associated co-morbidities. Further study of the pathogenesis, biological and clinical characteristics, and treatment of renal neuroblastoma is required to provide an optimal treatment for patients and to improve the patient's quality of life.
\end{abstract}

\section{Introduction}

Neuroblastoma is the most common extracranial solid tumor to occur during childhood. The cancer arises in the sympathetic

Correspondence to: Professor Qi-Lian Liang, Department of Oncology, Affiliated Hospital of Guangdong Medical College, 57 People Avenue, Zhanjiang, Guangdong 524001, P.R. China E-mail: lianqilian@gdmc.edu.cn

Key words: neuroblastoma, treatment nervous system and mainly affects the adrenal glands and retroperitoneum, with a distribution pattern that is similar in children and adults. In total, $>90 \%$ of the tumors are diagnosed in children $<10$ years old (1). The incidence in those $>20$ years old has been reported to be only 0.12 cases $/ 1$ million individuals (2). Renal neuroblastomas are extremely uncommon, particularly in adults. To the best of our knowledge, to date only one case of adult intrarenal neuroblastoma has been reported in the literature (3). The number of studies on the pathogenesis, biological characteristics, treatment and prognosis of the cancer have been limited due to this low incidence. The present study reports a case of left renal neuroblastoma in an adult patient with multiple bone and joint metastases, and discusses its clinical manifestations, diagnosis and treatment. Written informed consent was obtained from the patient's family.

\section{Case report}

In December 2013, a 22-year-old female was admitted to the Affiliated Hospital of Guangdong Medical College (Zhanjiang, China)with abdominal and joint pain that had been present for $>10$ days. There was no significant medical history. The patient had experienced no fever, chills, diarrhea, nausea, vomiting or hematuria. A physical examination revealed that the abduction, internal rotation and extension of the left shoulder and hip were limited. Computed tomography (CT) of the abdomen and pelvis showed a large heterogeneous and centrally necrotic mass, measuring $8.2 \times 7.3 \times 3.1 \mathrm{~cm}$, in the upper pole of the left kidney (Fig. 1A and B). A number of retroperitoneal lymph node shadows were apparent, along with multiple small, low-density lesions in the lumbar region. Round low-density nodules were also present in the liver. Additionally, the bone scan showed multiple bone and joint metastases (Fig. 1C).

The patient underwent a left nephroureterectomy and ipsilateral lymph node dissection. Pathological examination showed that the cells contained small, uniform, hyperchromatic nuclei, with coarse chromatin. Occasional larger ganglion-like cells were present and focal anaplasia was also evident. The 

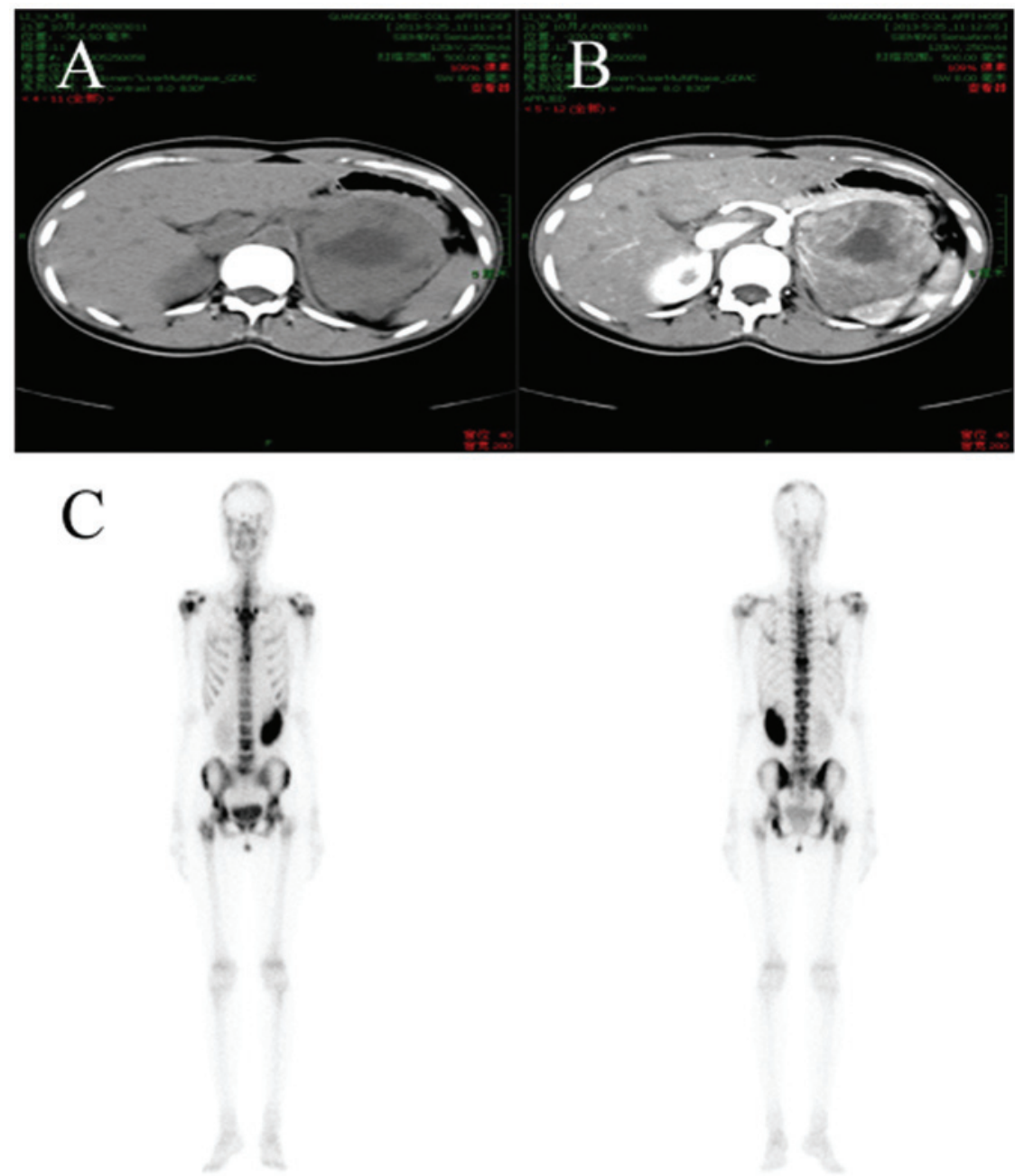

Figure 1. (A and B) Computed tomography scans of the abdomen and pelvis showing a large heterogeneous and centrally necrotic mass, measuring $8.2 \times 7.3 \times 3.1 \mathrm{~cm}$, in the upper pole of the left kidney. A number of retroperitoneal lymph node shadows were apparent along with multiple small low-density lesions in the lumbar region. Round nodules were also present in the liver. (C) Bone scan showing multiple bone and joint metastases.
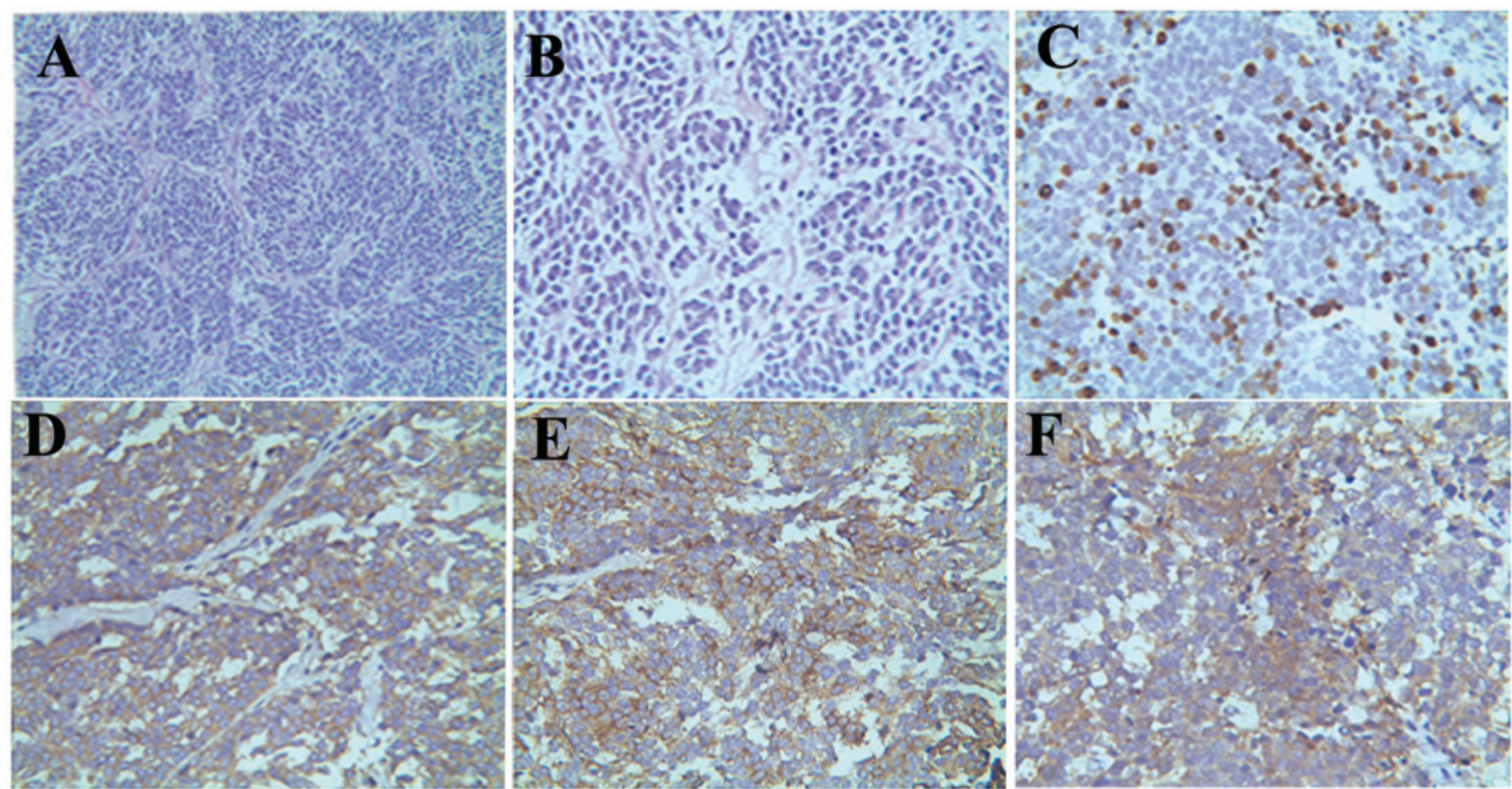

Figure 2. Pathological examination showed (A) cells containing small, uniform, hyperchromatic nuclei, with coarse chromatin (hematoxylin staining; original magnification, x200). (B) Occasional larger ganglion-like cells were present and focal anaplasia was also evident (hematoxylin staining; original magnification, $\mathrm{x} 400$ ). The tumor cells were strongly stained for (C) Ki-67 (original magnification, $\mathrm{x} 400$ ), (D) synaptophysin (original magnification, $\mathrm{x} 400$ ), (E) chromogranin (original magnification, $\mathrm{x} 400$ ) and (F) neuron-specific enolase (original magnification, $\mathrm{x} 400$ ). 
tumor cells stained strongly for chromogranin, synaptophysin, neuron-specific enolase, cluster of differentiation (CD)56 and vimentin, with a positive Ki-67 expression rate of $20 \%$. Focal dot negativity was exhibited for cytokeratin. Staining for epithelial membrane antigen, CK, leucocyte common antigen, S-100, CD10 and CD99 was negative in the renal tumor cells. These findings are consistent with renal neuroblastoma (Fig. 2). Post-operative radiotherapy (40 Gy) was administered for four weeks and the pain in the joints and abdomen consequently disappeared. The patient was discharged 60 days after admittance. At the nine-month follow-up examination, the patient showed no evidence of recurrence, progression or metastatic disease on CT scans of the chest, abdomen and pelvis.

\section{Discussion}

Neuroblastoma is the second most prevalent solid pediatric malignancy, with $>90 \%$ of tumors diagnosed in children $<10$ years old (1). To the best of our knowledge, the youngest recorded patient with a neuroblastoma was 30 weeks old (4) and the oldest patient was 79 years old (5). A recent study found that the incident rate of neuroblastoma $(80$ per 1 million individuals) ranked first highest and that the 5-year survival rate $(93.4 \%)$ was ranked third highest, amongst all types of cancer in the 309 cases of diagnosed cancer between 1998 and 2007 (6). Studies on the pathogenesis, biological characteristics, treatment and prognosis of renal neuroblastoma have been limited due to the low incidence of the cancer. At present, the pathogenesis of neuroblastoma remains extremely unclear. The pathogenesis of neuroblastoma may be associated with ALK, PTPN11, ATRX, MYCN, NRAS, CHEK2, PINK1 and BARD1 (7).

According to the current literature (8), the diagnosis of neuroblastoma depends mainly on ultrasound, CT, magnetic resonance imaging, post-operative pathology and fine-needle aspiration (FNA) cytology. For example, Serrano et al (9) diagnosed two primary renal neuroblastomas by CT-guided FNA cytology in a 6-year-old male and a 7-month-old male, thus demonstrating the usefulness of CT-guided FNA in diagnosing renal neuroblastoma. The treatment modalities for this cancer are surgery, surgery plus chemotherapy and surgery plus radiotherapy. A previous study has shown that ${ }^{177} \mathrm{Lu}$-DOTATATE could be used to treat children with relapsed or primary refractory high-risk neuroblastoma, and no significant renal toxicity has yet been observed (10). Due to the lack of specific treatment programs, clinicians do not have clear treatment guidelines to refer to and thus, they treated this patient according to pediatric guidelines. Tiu et al (5) presented one case of kidney neuroblastoma with unfavorable histological features that was treated with surgery alone. Long-term post-operative follow-up was also performed, which revealed no evidence to indicate recurrence. Bayrak et al (3) also reported one case in which a patient with stage I intrarenal neuroblastoma did not receive adjuvant therapy post-operatively; this patient also showed no evidence of recurrent residual disease at 30 months post-surgery. There is currently no evidence to show the difference between adjuvant chemotherapy and radiotherapy with regard to improving survival times and reducing recurrence.
Gohji et al (11) reported two cases of kidney neuroblastoma in two adult cases; one patient was treated with post-operative cobalt therapy and the other was treated with post-operative adjuvant chemotherapy. Upon comparison, no difference in tumor recurrence was found after a follow-up time of five years. Moreno et al (12) analyzed a large number of high-risk neuroblastoma survivors from a multi-institutional randomized trial that included the use of induction chemotherapy, surgery for the primary tumor and high-dose melphalan, with stem cell rescue. The study found no differences in the late toxicities between treatment arms for a median follow-up period of 12.9 years (range, 6.9-16.5 years). The literature shows no difference in the recurrence and late toxicities of post-operative adjuvant treatment. Perwein et al (13) found a five-year overall survival rate [mean \pm standard deviation (SD)] of $54.3 \pm 9 \%$ and a five-year event-free survival rate (mean $\pm \mathrm{SD}$ ) of $44.9 \pm 9 \%$ amongst 31 patients with stage IV neuroblastoma treated between 1984 and 2009. There was, however, a high incidence of severe long-term sequelae in the hepatobiliary, musculoskeletal and pulmonary systems, as well as unilateral blindness and secondary neurological neoplasms.

In the present study, CT of the abdomen and pelvis was used to locate the renal mass, while the bone scan showed multiple bone and joint metastases. The patient subsequently underwent a radical left nephroureterectomy and ipsilateral lymph node dissection. The post-operative pathology confirmed a diagnosis of renal neuroblastoma. The patient was also treated with post-operative radiotherapy, and nine months of follow-up revealed no significant side-effects or recurrence. For more detailed data, such as the five-year survival rate and the presence of any future recurrence, a longer follow-up is required.

In conclusion, renal neuroblastoma is extremely uncommon in adults, and its diagnosis and treatment is complicated by this low incidence, the lack of specific treatment guidelines, the advanced disease state due to late presentation, and its associated co-morbidities. Further study is required on the pathogenesis, biological and clinical characteristics, and treatment of renal neuroblastoma in order to provide an optimal treatment and to improve the patient's quality of life.

\section{References}

1. Franks LM, Bollen A, Seeger RC, et al: Neuroblastoma in adults and adolescents: an indolent course with poor survival. Cancer 79: 2028-2035, 1997.

2. Esiashvili N, Goodman M, Ward K, et al: Neuroblastoma in adults: incidence and survival analysis based on SEER data. Pediatric Blood Cancer 49: 41-46, 2007.

3. Bayrak O, Seckiner I, Erturhan S, et al: Adult intrarenal neuroblastoma presenting as renal cell carcinoma. Can Urol Assoc J 6: E144-E146, 2012.

4. Garnier S, Maillet O, Haouy S, et al: Prenatal intrarenal neuroblastoma mimicking a mesoblastic nephroma: a case report. J Pediatr Surg 47: e21-e23, 2012.

5. Tiu A, Latif E, Yau L, et al: Primary renal neuroblastoma in adults. Urology 82: 11-13, 2013.

6. Rabinowicz R, Barchana M, Liphshiz I, et al: Cancer incidence and survival among infants in Israel, 1998-2007. Pediatr Hematol Oncol 30: 646-654, 2013.

7. Pugh TJ, Morozova O, Attiyeh EF, et al: The genetic landscape of high-risk neuroblastoma. Nat Genet 45: 279-284, 2013.

8. Polishchuk AL, Dubois SG, Haas-Kogan D, et al: Response, survival and toxicity after iodine-131-metaiodobenzylguanidine therapy for neuroblastoma in pre-adolescents, adolescents and adults. Cancer 117: 4286-4293, 2011. 
9. Serrano R, Rodríguez-Peralto JL, De Orbe GG, et al: Intrarenal neuroblastoma diagnosed by fine-needle aspiration: a report of two cases. Diagn Cytopathol 27: 294-297, 2002.

10. Gains JE, Bomanji JB, Fersht NL, et al: 177Lu-DOTATATE molecular radiotherapy for childhood neuroblastoma. J Nucl Med 52: 1041-1047, 2011.

11. Gohji K, Nakanishi T, Hara I, et al: Two cases of primary neuroblastoma of the kidney in adults. J Urol 137: 966-968, 1987.
12. Moreno L, Vaidya SJ, Pinkerton CR, et al: European neuroblastoma study group; Children's Cancer and Leukaemia Group (CCLG) (formerly UKCCSG): Long-term follow-up of children with high-risk neuroblastoma: the ENSG5 trial experience. Pediatr Blood Cancer 60: 1135-1140, 2013.

13. Perwein T, Lackner H, Sovinz P, et al: Survival and late effects in children with stage 4 neuroblastoma. Pediatr Blood Cancer 57: 629-635, 2011. 